\title{
Transfer of wafer-scale graphene onto arbitrary substrates: The steps towards the reuse and recycle of the catalyst
}

Choon-Ming Seah ${ }^{1,2}$, Brigitte Vigolo ${ }^{2, *}$, Siang-Piao Chai ${ }^{3}$, and Abdul Rahman Mohamed ${ }^{1, *}$

${ }^{1}$ School of Chemical Engineering, Engineering Campus, Universiti Sains Malaysia, 14300

Nibong Tebal, Seberang Perai Selatan, P. Pinang, Malaysia

${ }^{2}$ Université de Lorraine, CNRS, Institut Jean Lamour, F-54000 Nancy, France

${ }^{3}$ Chemical Engineering Discipline, School of Engineering, Monash University, Jalan Lagoon Selatan, 46150 Bandar Sunway, Selangor, Malaysia 
*Corresponding authors:

Dr. Prof. Abdul Rahman Mohamed, chrahman@usm.my (A.R. Mohamed)

Dr. Brigitte Vigolo, Brigitte.Vigolo@univ-lorraine.fr (B. Vigolo)

\begin{abstract}
Graphene is a two-dimensional material, a single layer of carbon atoms with a honeycomb lattice, which has increasing demand, especially wafer-scale graphene for applications in electronics industry. However, graphene requires to be supported on different substrates depending on its utilization and the transfer stage must be achieved without damaging the graphene structure. Wet chemical etching is the major route for graphene transfer widely applied right now; the loss of catalyst during the separation process being the main limitation. Mechanical peeling is a simple process but the quality of the separated graphene remains poor. Currently, electrochemical delamination or bubbling method and water assisted delamination are new and most promising methods for both efficient graphene transfer and possible catalyst reuse. This article provides a comprehensive review of the various graphene transfer methods without compromise in catalyst deterioration and it concludes with the future challenges in the domain.
\end{abstract}

Keywords: graphene, graphene transfer, electrochemical delamination, water assisted graphene delamination catalyst reuse. 


\section{Introduction}

Graphene is a two-dimensional single layer of carbon atoms [1]. It is the fundamental block of the zero-dimensional fullerene, one-dimensional carbon nanotube and three-dimensional graphite and diamond. Graphene is an attractive material from the point of view of both science and engineering. Graphene is an amazing materials having outstanding electronic properties that are not shared by other materials [2,3].

The unique properties of graphene enable it to be applied in various applications. Various approaches have been developed to synthesize graphene, such as (1) top-down approach, i.e. mechanical exfoliation [2], ultrasound breaking of graphite [4], thinning of graphene with plasma [5] and reduction of graphene oxide [6], or (2) bottom-up approach, such as epitaxial growth of graphene from $\mathrm{SiC}$ [7] and chemical vapor deposition (CVD) of graphene by using metal catalysts, such as $\mathrm{Ni}[8]$ and $\mathrm{Cu}$ [9]. So far, the bottom-up approach is one of the most promising approaches to produce wafer-scale graphene with controllable size thickness and shape [10]. It is to note that both methods require the use of a substrate for the growth of graphene. The formed graphene is inevitably attached firmly on the substrate surface after growth. Due to the strong interactions between graphene and the underlying substrate, the electronic properties of graphene are strongly influenced and the symmetry of its honeycomb structure is highly distorted. The thermal, optical, mechanical properties graphene are severely hindered by the substrate. Because 
of that, graphene grown through CVD and SiC needs to be separated from the initial substrate to be used in practical applications.

In this review, the recent techniques for graphene transfer particularly developed to avoid excessive damage to the catalyst and graphene are discussed. The influence of substrate to graphene properties will start the article. This first part will be followed by the progress of various methods for the transfer of the grown graphene by CVD without deteriorating both graphene and catalyst; the essential advantage of such approaches is to envisage recycling the catalyst; it consequently induces to highly reduce of the graphene synthesis cost, for which the catalyst represents a high share of graphene production cost. Additionally, the advantages and shortcomings of each method and the modifications made are reviewed critically. Finally, the future research activities, directions, and challenges to improve the separation process and the up-scaling of the transfer method and reuse of catalyst to meet the requirements for industry are summarized and wrap up the article.

\section{Influence of the substrate on the graphene properties}

The monolayer thickness of graphene is the main source of its unique properties but also the cause of many issues associated with graphene. Graphene exposes majority of its atoms to the surrounding environment. It easily forms dangling bonds with the supporting substrate, resulting in inhomogeneities of charge which alters the conduction path of electrons and holes. Furthermore, graphene that adheres firmly to the substrate always adopts the surface morphology of the substrate. This feature creates undesired strain and further scatters the path of electron diffusion [11-13]. For the synthesis from $\mathrm{SiC}$ sublimation, graphene is sometimes even considered as covalently bonded to the $\mathrm{SiC}$ substrate. The first layer of graphene is then referred 
as the buffer layer without graphene characteristics [14]. The distance between the planar SiC (0001) surface and the first carbon layer was measured to be $2.62 \pm 0.13 \AA$, the short distance is contributed by the a partially sp3-hybridized graphene buffer layer due to the strong bonding with the outmost $\mathrm{Si}$ atoms of the SiC substrate [15]. This distance being much smaller than the interlayer spacing of graphite $(\sim 3.3 \AA)$ induces distortion of the Fermi level to above the Dirac point; graphene behaving like a weak n-type semiconductor with a narrow band gap. This behavior is a result of the upward shift in the Fermi level from the Dirac point due to the dangling bonds on the SiC surface [16]. The intrinsic doping effect may persistently influence more than one layer of graphene [17]. The band gap is approximately $0.26 \mathrm{eV}$ for monolayer graphene, and it decreases with an increase in the number of graphene layers [18]. However, it has also been reported that the electronic properties of 10-layergraphene on $\mathrm{SiC}$ is similar to one single layer graphene [19].

The strong coupling effect between graphene and $\mathrm{SiC}$ results in temperature dependence of the conductance of graphene [20]. The highest electron mobility of graphene on SiC ever recorded is $150,000 \mathrm{~cm}^{2} \mathrm{~V}^{-1} \mathrm{~s}^{-1}$ [21] on a C-terminated surface, basically was caused by the misorientation due to relatively weaker coupling between graphene and C-terminated surface, but the majority of the articles report that mobility in the range of $500-5000 \mathrm{~cm}^{2} \mathrm{~V}^{-1} \mathrm{~s}^{-1}$ under various conditions $[20,22-25]$; it is much lower than the mobility calculated for free standing graphene, i.e. $200,000 \mathrm{~cm}^{2} \mathrm{~V}^{-1} \mathrm{~s}^{-1}$ [26]. And the charge carrier density is in range of $10^{11}$ to $10^{14}$ $\mathrm{cm}^{-2}$. The change in charge density, due to the strong interactions of graphene with SiC, highly limits its application in devices due to gating difficulties [27]. These changes in properties were only found in graphene directly grown on $\mathrm{SiC}$ and not in graphene physically deposited on $\mathrm{SiC}$ [28]. 
Graphene grown by CVD also has its own limitations. The measurements of the transport properties of graphene grown on transition metal catalysts also deviate depending on the nature of the used metal substrate because transition metals have their own electrical and transport properties. The empty d-shell of the transition metals induces hybridization between the graphene $\pi$-band and its d-valance band. It has been reported that this strong interaction would alter the electronic properties up to the second layer of graphene grown on $\mathrm{Ru}[29,30]$. For $\mathrm{Ni}$ catalyst, the $\pi$-band structure of graphene could not be found in the first two layers [31]. Ni could severely modify the electronic structure of graphene leading to appearance of an effective magnetic moment of carbon atoms in the graphene layer, where unpaired electrons exist [32]. Furthermore, alteration of the electronic properties of graphene may disrupt ballistic transport in graphene. However, this change provides an alternative route of tunneling magneto-resistance and gives a solution for this obstacle in the spintronic field [33].Transition metals could cause a "hole drilling" effect on the graphene sheet. It is known that transition metals, such as $\mathrm{Cu}, \mathrm{Ni}$, $\mathrm{Co}$ are catalytically active to grow graphene. On the other hand, they have the ability to etch graphene under high temperatures $[34,35]$. Although the electrical and mechanical properties of graphene are affected by contact with transition metals, the strong interactions between them are also beneficial if the structure is used as the gate electrode in graphene-based electronic devices [36].

The industrial value of graphene grown either by CVD or through sublimation of SiC, Although graphene on $\mathrm{SiC}$ can be applied in various electronic devices, such as field effect transistors (FET), radio frequency (RF) transistors, integrated circuits (IC), and sensors [37]. But the application is still limited due to the interactions between graphene and its original substrate (transition metals or $\mathrm{SiC}$ ). The relocation of wafer-scale graphene onto a suitable substrate is 
vital to conserve the unique properties of graphene and is a critical issue that needs to be addressed and solved [10]. Deposition of graphene onto insulating materials is necessary for electronic applications [38]. For example, graphene on a transparent substrate can serve as a transparent conductor, and graphene on a $\mathrm{SiO}_{\mathrm{x}}$ substrate can be incorporated in Si-based devices. However, graphene deposited on $\mathrm{SiO}_{2}$ encounters problems of inhomogeneous distribution of charges due to a hole doping effect. On the contrary, graphene on Pt preserves its free-standing properties, and Pt can act as a mechanical support for graphene [39]. And by adding a layer of $\mathrm{Y}_{2} \mathrm{O}_{3}$, a high k-dielectric onto graphene/Pt, the formed dielectric layers could be used for charge doping of metal-supported graphene [40]. Hexagonal boron nitride has a similar structure to that of graphene. The surface topography of graphene can be better preserved as it is placed onto boron nitride. Furthermore, a clean surface of thin-layer boron nitride is free from dangling bonds and the electrical properties of graphene are hence well maintained as graphene is deposited on a layer of boron nitride [41, 42]. A polymer film such as polyethylene terephthalate (PET) has been shown to be a good substrate for graphene. It was reported to cause no degradation on its electronic properties because of the polymer nature of such substrate which has very low interactions with the electronic cloud of graphene [43]. Meanwhile, Ge(001) substrate was also reported to enhance the charge transport property of graphene. The charge mobility is of $\sim 5 \times 105 \mathrm{~cm}^{2} \mathrm{~V}^{-1} \mathrm{~s}^{-1}$ at $20 \mathrm{~K}$, and the carrier density in the graphene is as high as $10^{14} \mathrm{~cm}^{-2}$ at $300 \mathrm{~K}[44]$.

The superlative transfer and positioning of wafer-scale graphene on arbitrary substrate) without causing damages to its crystallinity is a challenging task and it remains crucial for the development of new graphene-based devices. At present, wet chemical etching of the substrate is the dominant transfer method applied to graphene in most of articles. However, such method 
usually implies a complete consumption of the metal catalyst, incurring higher cost for producing graphene. Furthermore, chemical etching generates the large quantity of chemical wastes which require vigilant disposal because the metal residues coming either from catalyst or from the etchant, even in very small amount under the form of trace, severely alter the electronic, optical and mechanical properties of the graphene material $[45,46]$. Hence the exploration on the graphene transfer methods which could preserve the perfectness of the surface of catalyst foil but without deterioration of the crystallinity of graphene is important and it represents the major branch of graphene research area right now.

\section{Mechanical exfoliation}

The first application of mechanical exfoliation method for graphene separation was reported by Novoselov et al., who used scotch tape to exfoliate graphene form highly oriented pyrolytic carbon (HOPG) [2]. Mechanical exfoliation is a straightforward transfer process. It is one of the most popular approaches for wafer-scale graphene transfer, after the wet chemical etching transfer method. For the wafer-scale graphene transfer, this method was first applied to transfer graphene prepared by $\mathrm{SiC}$ sublimation [47]. Kim et al. [48] demonstrated exfoliation of graphene from $\mathrm{SiC}$ via the stress induced with a Ni film. Subsequently, the excess graphene is selectively removed with a second exfoliation process with a Au film to ensure that a monolayer graphene is obtained. The author also demonstrated that the SiC can be reused again for CVD to obtain graphene with the same quality after up to 5 cycles. After all, mechanical exfoliation was more often applied on transfer of wafer-scale graphene grown from $\mathrm{Cu}$. The adhesion energy between graphene and $\mathrm{Cu}$ was measured experimentally to be around $0.51 \mathrm{~J} . \mathrm{m}^{-2}-0.72 \mathrm{~J} . \mathrm{m}^{-2}$. Hence, the transfer agent just needs to have stronger adhesion energy towards graphene to allow graphene 
pealing from $\mathrm{Cu}[49,50]$. Particularly, $\mathrm{Ni}$ was calculated to have the adhesion energy about 3.47 $\mathrm{J}^{-\mathrm{m}^{-2}}[50]$ with graphene; that makes the peeling of graphene from Ni without deterioration very challenging. On the other hand, hydrogen-terminated germanium is an expensive catalyst for graphene CVD, but it has very weak coupling with graphene. Mechanical exfoliation is an ideal transfer method, since it involves no chemical process that may deteriorate Ge substrate. Peeling of graphene from Ge is found to be very simple and allows multiple uses of the same Ge foil as catalyst without degradation of the formed graphene [51].

The graphene transfer by mechanical exfoliation is one of the earliest methods for waferscale graphene separation and it has been already widely reviewed $[52,53]$. The main advantage of mechanical exfoliation is the minimum damaging created to the surface of the catalyst foil. The graphene sheet could be directly peeled off from the metal foil by using an adhesion film that provides strong adhesion forces, higher than the interaction between graphene and the metal substrate. The transferring agent could be as simple as Thermal Release Tape (TRT), polymer [49] and hexagonal boron nitride [54] or could be as complex as introduction of organic linker [55] (Fig. 1) or heavy metal as adhesive [56]. For transfer printing, a simple polydimethylsiloxane (PDMS) film can be used as an adhesive tape to peel off the graphene [57]. In this method, liquid PDMS is directly coated onto the graphene/catalyst. After the polymer is dried and hardened, the PDMS/catalyst film is ready for peeling to separate the graphene from the metal substrate. The number of graphene layers to be peeled off can be controlled by adjusting the ratio of PDMS to the curing agent. Another simple transfer process was reported by Yoo et al. [58]. A PDMS stamp was prepared after mixture with the curing agent. The dried film was then pressed directly onto a metal foil with graphene and exfoliated, rather than coated on graphene. Exfoliation of graphene from a metal substrate with a rod shape was also demonstrated 
by $\mathrm{Su}$ et al. [59]. PDMS has also been utilized by researchers to exfoliate graphene from HOPG or for using in the transfer printing of patterned graphene [60]. However, the PDMS stamp transfer method is efficient if the adhesion energy between graphene and the desired substrate is strong enough. The PDMS stamp transfer is only useful for transferring graphene to flat and hydrophilic substrates, and cracks formed during the transfer process are often observed [61].
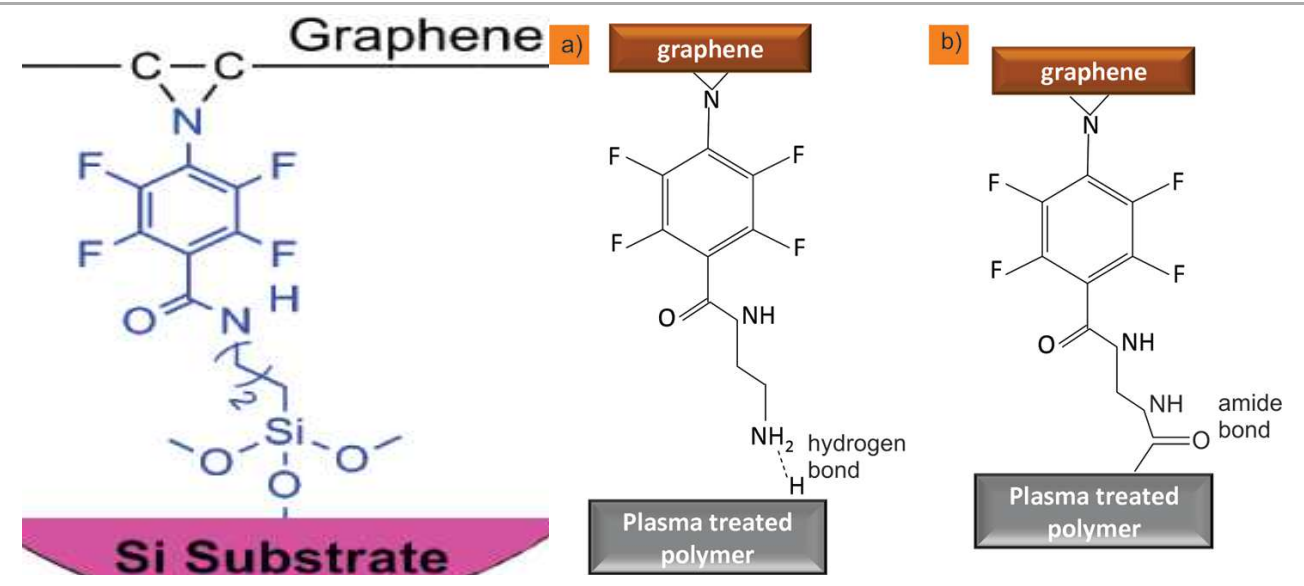

Figure 1:(a) Immobilized graphene on silicon wafer via the PFPA-Silane Coupling Agent [62] . (b) \& (c) TFPA attachment on plasma treated PS film with hydrogen bonding with covalent amide bond [63]. Copyright 2012 American Chemical Society.

Under the mechanical exfoliation process, the metal foil is normally unharmed and ready to be reused again for a subsequent CVD growth for graphene formation. However, the study toward reuse and recycling of the catalyst foil is rarely found. The damage induced to the graphene during the mechanical transfer process, is normally caused by an inappropriate contact between the graphene sheet and the polymer or the adhesive tape. The adhesion force applied by the used transfer agent is always not distributed evenly throughout the graphene surface. Thus, large voids, tears and wrinkles are often observed in the graphene sheet after transfer. 
The polymer assisted transfer possesses a critical weakness since it is difficult to transfer graphene over very large areas. A special process has been proposed: the "roll-to-roll" (R2R) can be used to transfer 30-inch graphene films [64]. The process is illustrated in Fig 2. It consists in 3 main steps: (i) adhesion of the thermal-released tape as a polymer support onto the graphene/metal film, (ii) etching of the metal and (iii) release of the graphene from the tape and deposit onto the chosen substrate. The discovery of the R2R process has opened a new chapter for the graphene synthesis and transfer studies. R2R has been adapted by researchers to design the continuous synthesis of graphene through CVD $[65,66]$. The original design utilized a wet chemical etching approach for graphene separation. Juang et al. had further been modified it to let the separation done mechanically from $\mathrm{Ni}$ catalyst preserving the catalyst. However, the graphene broke into pieces after the separation process, the properties were consequently significantly lost. Line-shaped cracks were shown to propagate perpendicular to the roll direction and voids of micron size were formed on graphene [67].

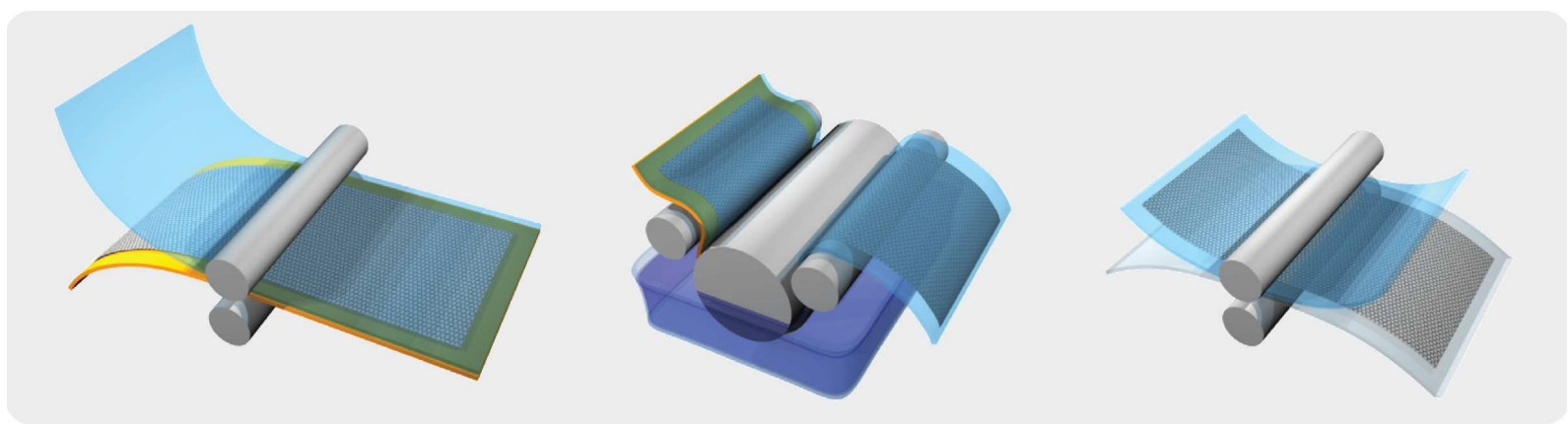

Figure 2: Schematic of the roll-based production of graphene films grown on a copper foil. It starts with the adhesion of polymer support onto the graphene/Cu through rolling compression. The polymer/graphene/ $\mathrm{Cu}$ is subsequently rolled over the etchant to etch away the $\mathrm{Cu}$ foil. Eventually, the remaining polymer/graphene will be compressively rolled with the target substrate to release graphene from polymer to target substrate and complete the R2R process [64]. Copyright 2010 Nature Publishing Group. 
Mechanical exfoliation is a simple and reliable method allowing recycling the metal catalyst. It is very important for the mass production of graphene at lower cost. But the main drawback remains the introduced large scale physical defects poised onto the plane of the graphene sheet. And, until now, recycling the catalyst through mechanical exfoliation was rarely investigated in the literature. And alternative methods are developed in order to envisage the possibility of reusing the catalyst substrate.

\section{Electrochemical delamination}

Electrochemical delamination, also known as bubbling transfer method is one of the most innovative wafer-scale graphene transfer method that has been introduced to enable the reuse of the catalyst foil. The research group from National University of Singapore who first reported the reuse of a $\mathrm{Cu}$ foil of thickness of $25 \mu \mathrm{m}$ up to 3 times. Previously, the electrochemical delamination method was more often applied to exfoliate graphene flakes from bulk graphite [68]. Polar solvent or polaraprotic solvent, such as methyl-imidazolium hexafluorophosphate [69] is used as electrolyte to intercalate into inter planar spaces of graphite to break it into graphene flakes with single of few layers.

Basically, the separation of wafer-scale graphene by electrochemical delamination method implies the use of an electrochemical cell to allow the graphene separation from respective catalyst foil. For example, a simple electrolysis cell with a constant current supply is applied to the catalyst foil and leads to dissociate the water molecules into oxygen and hydrogen. Those gases are playing the role in weakening the coupling between graphene and catalyst for the separation process [70]. The electrochemical exfoliation is a "wet" process which involves deposit of a layer of poly methyl methacrylate (PMMA) that prevents graphene folding. This 
polymer layer could also serve as a protective layer for graphene from physical damages from turbulences formed within the liquid during formation of the hydrogen bubbles. A typical setup of electrochemical delamination is shown in Fig. 3 [70]. Oxygen ions were reported to possess the ability to permeate into interface in between $\mathrm{Cu}$ and graphene, especially at the wrinkles of graphene [71]. Protons (hydrogen ions), smaller in size, were found to have a better capability to permeate through graphene to the interface between graphene and metal catalyst [72]. The current applied at the catalyst foil induces the reduction of protons to form numerous hydrogen bubbles which subsequently abolish interactions between the graphene sheet and the metal catalyst and lead to detachment of the graphene sheet from the metal foil [73, 74]. It was even reported that, protons were able to penetrate through both thin film of PMMA and graphene, to allow isotropic graphene delamination [75]. But another view pointed out that the hydrogen bubbles only formed at the cavities near wrinkles of graphene [76]. Main advantage of electrochemical delamination is the preservation of the flatness of the catalyst surface; avoidance of chemical attack prevents the catalyst surface to be corrugated. The flatness of catalyst surface is important to obtain graphene uniform in thickness; graphene indeed fully copies the topography of the catalyst surface during the CVD growth, and a corrugated catalyst surface will give inflated graphene [73, 77]. 


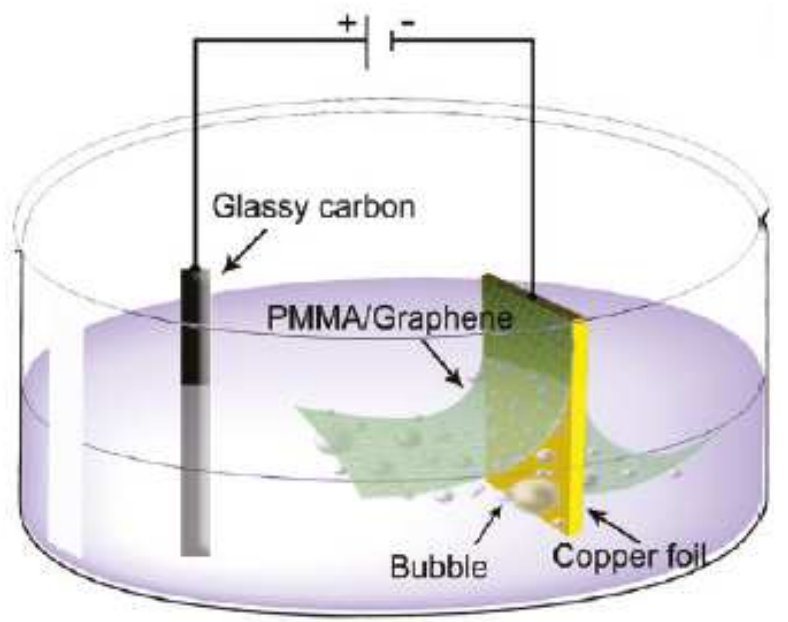

Figure 3: Schematic of an electrolysis cell used for the electrochemical exfoliation of graphene from metal foil. A direct current is applied to facilitate the electrolysis process to form hydrogen bubbles at the surface of $\mathrm{Cu}$ through reduction of water, and to delaminate the graphene from $\mathrm{Cu}$ [73]. Copyright 2011 American Chemical Society.

In general, the rate of graphene delaminated electrochemically depends on the concentration of electrolyte, the conductivity of electrolyte, the potential difference applied, and the type of electrolyte. Among all the factors, the type of electrolyte is the dominant factor since the conductivity of solution highly depends on it [78]. In most of the occasion, basic solution, such as sodium and potassium salts are utilized for rapid electrochemical delamination. The redox process that normally happens within acidic solutions and leads to etch the catalyst is hence avoided. Basic electrolytes minimize indeed consumption of the transition metal catalyst foil during the separation process. Furthermore, the rate of graphene delamination is noteworthy much faster by using a basic-type electrolyte as compared with acidic electrolyte [79]. Fundamentally, the electrochemical delamination separation process can be understood based on the water electrolysis. The minimum potential difference that needs to be applied between cathode (normally the catalyst foil with graphene) and anode (other inert metal, i.e. $\mathrm{Au}, \mathrm{Ag}, \mathrm{Pt}$ and graphite) is $\sim 1.229 \mathrm{~V}$ at room temperature, close to that used for electrolysis of water. An 
increase of the potential difference applied between the 2 electrodes leads to accelerate the delamination process [80]. In bubbling process, water has to be intercalated in between graphene and metal substrate. It is allowing the bubbles to be formed in between graphene and metal substrate, so that the delamination process would be efficient. Under high potential difference, the rate of water intercalation would be the rate limiting factor, because the water intercalation is a relatively slow process. The rate of delamination of graphene from the metal substrate is not increased significantly with very high voltage $(\sim 10 \mathrm{~V})$, since most of the bubbles are not formed in between graphene and metal substrate [81]. In some occasion, a relatively small voltage could also be applied to oxidize the $\mathrm{Cu}$ surface and to weaken its interaction with graphene; the electrolyte providing free cations and anions and facilitating the electrolysis phenomenon to take place smoothly. Pt and Ir are relative inert metal, application of small voltage $(<1 \mathrm{~V})$ will not oxide the surface of $\mathrm{Pt}$ and Ir. The water molecule intercalates into the interface between graphene and Pt or Ir to form layer of platinum hydroxide or iridium hydroxide, instead of oxide. Hydrogen bubbles, also generated under small voltage, but the volume is too small to allow the delamination [82]. The voltage applied has to be higher to kick start the delamination process of graphene from Pt or Ir.

The concentration, conductivity and the type of electrolyte are the main factors that control the rate of graphene delamination [79]. Low concentration of electrolyte is reported to lead to an incomplete separation of graphene from the catalyst foil mainly due to the slow and uneven hydrogen bubble formation across the metal catalyst surface [83]. Vice versa, the rate of delamination increases with increasing the electrolyte concentration [76]. A high concentration of electrolyte can secure a smooth separation process [84]. But over high concentration of electrolyte may result in a large scale of residues deposited on the graphene basal plane after 
separation [85]. On the other hand, the use of an aqueous solution with a better electrical conductivity is shown to assist the rate of bubble formation and the rate of graphene delamination [86]. However, a vigorous separation rate can induce deterioration of the graphene sheet. The effect of the different nature of electrolyte on the graphene delamination remains unconcluded. There is no significant deviation in the quality of delaminated graphene by utilizing different types of basic electrolytes [87]. The degradation of the graphene properties mainly depends on the rate of delamination, through a more vigorous hydrogen bubble formation leading to large structural defects. The electrolysis process happens at both surfaces of metal. If one of the surface is totally shielded from the electrolyte, it was reported that the other side possesses the ability to accelerate the graphene delamination rate [88].

Since after the electrochemical delamination process was proposed, numerous reports have been published for further improvements and modifications of the process, with the purpose of a better preservation of the quality of graphene and reusability of the catalyst foil. The perfectness of the graphene sheet can be simply protected by using a thick coating layer of PMMA which is a rigid support minimizing the mechanical damage [75]. However, PMMA can be difficult to be removed afterwards. An additional layer of protective film was suggested to counter deterioration during separation. For example, a polyethylene terephthalate (PET) or thermal release tape (TRT) or conventionally available screen protectors for handset can minimize physical damages induced to graphene $[76,85,89]$. Somehow, a more rigid polymer frame could also be applied at the perimeter of the PMMA coated on graphene. It could provide stronger support and reduce the damage caused by bubbles, especially at the edges of graphene more vulnerable to be damaged $[84,85]$. Sun et al. [90] noticed that protons are able to permeate through the PMMA film. A small amount of hydrogen molecules is believed to form at the 
interface of PMMA and graphene, resulting in void nucleation between graphene and PMMA, and finally leading to hole formation on graphene. It was hence proposed to encapsulate the air gap between graphene and PMMA as a permeation stopping layer, preventing the uncontrollable void formation [90]. Meanwhile, for the application of graphene in certain fields such as transparent conductive film, flexible polymer based-substrate could be directly used as a supporting film for graphene. Avoiding the usage of PMMA is beneficial since its removal implies a tiresome process. Furthermore, residues of PMMA severely degrade the electronic properties of graphene $[83,91]$.

Instead of metal catalyst foils, graphene also can be grown by CVD by using a sputtered metallic film onto silicon wafer, which is having thickness in nanoscale. The separation of graphene from thin catalyst film is much more complicated. Under such circumstances, the catalyst film is usually in direct contact with a silicon wafer, quartz, and other substrate to form graphene. There are 2 possibilities for the hydrogen bubble formation, they are either formed at the interface of $\mathrm{Cu}$ and silicon [92] or between $\mathrm{Cu}$ and graphene [93]. If hydrogen forms at the interface between $\mathrm{Cu}$ and silicon, the graphene is still firmly attached to $\mathrm{Cu}$. At the end of the process, $\mathrm{Cu}$ needs to be etched away to obtain clean graphene. Chromium could be added in between $\mathrm{Cu}$ and the sapphire substrate in order to secure that hydrogen bubbles only form in between graphene and $\mathrm{Cu}$. The catalyst was reported to be reused up to 7 times with minimum variation in quality of graphene [94]. But there is no extensive study that has been carried out to confirm the factor leading to formation of bubble either at the interface between $\mathrm{Cu}$ and silicon or interface between graphene and $\mathrm{Cu}$.

The design of the electrochemical cell was extensively improved to allow a more reliable separation process. A bi-electrode electrochemical transfer process was proposed by Shi et al. 
[95]. The PMMA/graphene/Cu stacks were used as both cathode and anode to allow simultaneous separation of 2 graphene sheets. And the PMMA/graphene/Cu stack placed at anode underwent the conventional wet chemical process while electrochemical delamination took place at cathode. However, the quality of the separated graphene was relatively poor. A two-step method has also been designed to permit a better lift-off of graphene from the metal catalyst. A lower potential was applied at the first stage to allow the protons to intercalate between the graphene sheet and the metal catalyst for tens of minutes, through under-potential deposition of protons. Intercalation of protons merely weaken the interfacial forces and facilitate the second stage. The potential difference steeply increases to kick start the evolution of hydrogen bubbles and lift-off the graphene from the metal substrate [80]. Koefoed et al. further improved such 2 step-process [96]. By taking advantage on the relatively weaker coupling of graphene with Ir used as catalyst, the authors have ambitiously intercalated large tetraoctyl ammonia ions $\left(\mathrm{TOA}^{+}\right)$between Ir and graphene. In the subsequent second step, delamination of graphene occurred within seconds. In the later study [97], the author managed to intercalate tetraalkylammonia ions into interface between graphene and $\mathrm{Pt}$ and $\mathrm{Cu}$, where the spacing between graphene graphene and $\mathrm{Cu}$ is much smaller compared with the spacing between graphene and Ir. Recently, an additional step was applied. Cesium (Cs) was first intercalated at the interface between graphene and Ir. Cs could barely lift graphene from Ir, which allowed under potential deposition of protons and complete delamination [98]. It was found that intercalation of Cs significantly reduces damaging on graphene during separation. Deng et al. enhanced the R2R method with electrochemical delamination to avoid the peeling process that normally imposes large scale mechanical defects to the separated graphene sheet. The authors also claimed that the $\mathrm{Cu}$ foil they used was highly preserved and could be reused 3 times [99]. 
Electrochemical delamination has also been demonstrated to efficiently separate graphene flakes grown on $\mathrm{Cu}$ powder. Due to the hydrophobic nature of graphene, the bubbles formed during the electrochemical delamination process attach to the graphene flake and float to the surface of water assisting that way the separation of the graphene flake from $\mathrm{Cu}$ [100].

Formation of turbulences during the formation of hydrogen bubbles is the main factor causing physical damages on the transferred graphene. Bubbles formed by electrochemical delamination are suggested to minimize the defects. The under potential delamination is only applicable on relatively inert metals (Ir and $\mathrm{Ru}$ ) but the behavior is different from that observed with $\mathrm{Cu}$ of higher electro-positivity. Application of a potential difference lower than the threshold of water electrolysis is expected to oxidize the surface of $\mathrm{Cu}$ to form $\mathrm{Cu}_{2} \mathrm{O}$, diminishing that way the coupling forces between graphene and its substrate. Subsequently, water will intercalate between $\mathrm{Cu}$ and graphene to complete delamination [101]. Somehow, the sample is immersed into the electrolyte gradually to allow delamination. $\mathrm{Cu}_{2} \mathrm{O}$ is formed after contact with the electrolyte medium and such method reduces strain on graphene [102]. It was reported that the binding energy between graphene and $\mathrm{Cu}$ substrate could reduce by $33 \%$ with the introduction of cuprous oxide [103]. Cu foil is readily to be reused again after reduction of the oxide layer. The delamination of graphene only starts after contact with electrolyte. Wang et al. further improve the electrochemical delamination process to become "bubble-free". A thin layer of $\mathrm{Cu}_{2} \mathrm{O}$ was purposely formed at the interface between graphene and copper foil. The continuous supply of electric current and soaking in weak acidic electrolyte, is facilitating dissolution of $\mathrm{Cu}_{2} \mathrm{O}$ without forming bubbles. The copper foil after delamination is free of copper oxide and ready for a subsequent graphene synthesis [104]. The roll-to roll (R2R) method can be improved with the implement of electrochemical bubbling delamination. The 
graphene/catalyst is rolled into electrolyte to slowly initiate the partial bubbling process. By this method, the delamination rate is faster and easier to be controlled, as compared with conventional R2R method that may result in total loss of catalyst [79]. Tungsten is another catalyst seldom used for graphene formation. It was reported that, graphene could be delaminated with hydrogen bubbles [105]

The bubbling delamination has also been demonstrated without utilization of the electrochemical method. Instead of using the electrical current to generate bubbles, the authors used purely chemical reaction for the bubble generation. From direct immerse of PMMA/graphene/Cu stack into Standard Clean Solution 1 (mixture of ammonium solution with hydrogen peroxide), oxygen bubbles are generated during the decomposition of hydrogen peroxide and intercalate between $\mathrm{Cu}$ and graphene. It is leading to lift-off the graphene from $\mathrm{Cu}$. This method is simple but it scarifies the metal catalyst [106]. On the other hand, some researchers operate this approach in a reversed way to enhance the catalyst etching by using the catalyst foil as anode to etch it electrochemically. This approach gives a much cleaner and efficient wet chemical etching for graphene separation [70, 107]. Instead of applying a potential difference, Choi et al. separated graphene from Pt through bubbles formed by boiling a solution of $\mathrm{NaOH}$ at high concentration $(0.5-4 \mathrm{M})$; the separation time ranging from 1.5 hours $(0.5 \mathrm{M}$ $\mathrm{NaOH})$ to roughly 0.5 hour $(4 \mathrm{M} \mathrm{NaOH})$. Under heating, $\mathrm{NaOH}$ hydrolyzed the Pt surface to facilitate the graphene separation, however Pt is a relatively inert metal, the chemical reaction was very minor and did not give much defects to the surface of Pt after separation, allowing it to be reused again. The authors also claimed that a heat-assisted bubbling method could better preserve the quality of the transferred graphene by reducing wrinkles and tears during the bubbling process [108]. 
Potential difference has been employed for electrostatic transfer of graphene from $\mathrm{SiC}$ or a metal foil catalyst. This approach takes advantage from the anionic bonding between the metal and glass substrate with mobile alkali cations. The negative charges produce an electric field and pull the graphene/SiC substrate. The anodic bonding facilitates a firm interaction between glass and $\mathrm{SiC}$ and favors the graphene transfer [109]. The firm bond is shown to minimize the voids or trapped air or moisture between graphene and glass [110].

The mechano-electro-thermal (MET) process is a new chemical-free alternative [111] (Fig.4). An electric field is used to exfoliate graphene from $\mathrm{Cu}$, the high physical pressure created allows the graphene sheet to have maximum contact with the substrate and the induced increase of temperature increases the visco-elasticity of a targeted substrate which could adhere to graphene. From the combination of these 3 forces (mechanical, electric and thermal), strong and ultra-conformal contact between the graphene and the target substrate can be achieved. Graphene is hence able to exfoliate with minimum physical damages and without chemical contamination.

The surface smoothness of metal catalyst is crucial to form high quality graphene [77]. Mutlu et al. [112] utilized the electrolysis cell (Fig. 3) for another purpose, instead of delamination of graphene. By using acidic solution, such as sulphuric acid or phosphoric acid as electrolyte, the electrolysis cell can be used to electro-polish the surface of $\mathrm{Cu}$ before CVD process to synthesis graphene. The graphene that formed of smooth surface is easier to be delaminated from $\mathrm{Cu}$ in subsequent electrochemical delamination process to obtain contamination free graphene. 


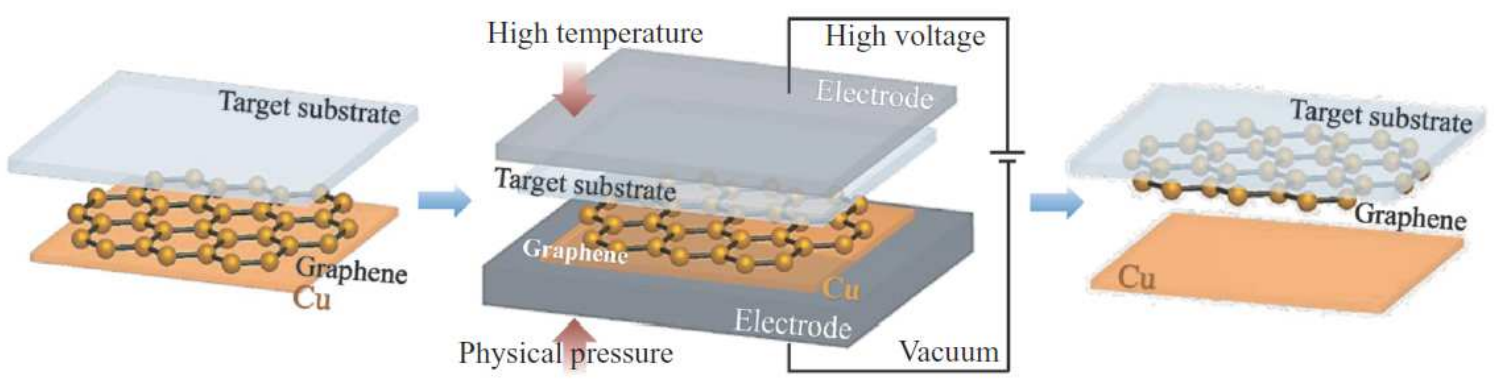

Figure 4: Schematic of graphene separation through the MET process avoiding deterioration of the $\mathrm{Cu}$ catalyst proposed by Jung et al. Mechanical pressing is first applied to align graphene/Cu and target subarea. Subsequently, electrostatic force is applied across graphene/Cu and target substrate, under high vacuum and thermal treatment to adhere graphene onto target substrate. The graphene/target substrate could be peeled away from $\mathrm{Cu}$ easily [111]. Copyright 2010 American Institute of Physics.

Electrochemical delamination is undoubtedly one of the best approaches enabling effective separation of graphene without deterioration of both metal catalyst and graphene. It breaks the bottleneck of graphene synthesis research which utilizes relatively inert transition metals, i.e. Ir, $\mathrm{Ru}$ and $\mathrm{Pt}$ as catalyst. These latter are hard to be separated from graphene by conventional wet-chemical etching methods. The electrochemical delamination process allows a very fast separation of graphene and the separated graphene is free from residue transition metal that may create parasitic effects. Graphene shows better electron transfer as compared with that separated by wet-chemical etching. Electrochemical delamination is the most developed approach to envisage the reuse of catalyst foil for graphene formed by CVD, however there still are a few shortcomings. Graphene separated through electrochemical delamination secures a better cleanliness; iron salt solutions used as etching agents kill the electromagnetic transport characteristics of graphene [113]. However, the relative vigorous separation mechanism induced by bubbling phenomenon requires the usage of a polymer film such as PMMA in order to protect graphene against mechanical damaging. And PMMA is another source of contamination which 
was shown to degrade the properties of graphene [91]. Using of high potential difference was suspected to negatively charge the graphene which can react with PMMA and render its removal difficult [96]. Other than that, most of the success cases of graphene separation by delamination are performed from thick catalyst foil. For separation of graphene formed on very thin (nanoscale) catalyst, sputtered onto silicon wafer for example, the bubble formation is favored at the catalyst/silicon interface without efficiency to separate graphene. The catalyst remains firmly attached to graphene requiring another catalyst etching process [93].

To date, graphene has been reported to be successfully delaminated from $\mathrm{Cu}, \mathrm{Pt}, \mathrm{Ru}, \mathrm{Ir}$ and $\mathrm{W}$ [105]. $\mathrm{Cu}, \mathrm{Pt}, \mathrm{Ru}, \mathrm{Ir}$ and $\mathrm{W}$ catalysts have a relatively weak coupling with graphene and spacing between graphene. For example, $\mathrm{Cu}$ and $\mathrm{Pt}$ are reported to range between $2.24 \AA-3.58$ $\AA$ [114] and $2.4 \AA$ - $3.7 \AA$ [115], respectively. The large spacing between weaken the coupling force between graphene and metal substrate. On the other hand, the spacing between $\mathrm{Ni}$ and graphene is much closer, which around $2.11 \pm 0.07 \AA$ [116]. The small spacing and strong coupling force between graphene and nickel making the delamination of graphene from Ni very hard. $\mathrm{Ni}$ is the most widely applied catalyst for graphene growth after $\mathrm{Cu}$. To date, only multilayer graphene could be separated from Ni by electrochemical delamination [117]. The size of a water molecule is around $3 \AA$, which means that water cannot be inserted between graphene and $\mathrm{Ni}$, while the spacing between graphene and $\mathrm{Cu}$ or $\mathrm{Pt}$ is large enough for water molecules to intercalate into the space between them. Besides, comparisons made by some researchers show that the amount of metallic residues on graphene separated by conventional wet etching methods is similar to that obtained through electrochemical delamination [118]. The sheet resistance of graphene delaminated electrochemically was found to be higher compared with that of graphene separated by standard chemical etching [87]. But an opposite view claims that electrochemical 
delamination gives a metal-free graphene after transfer process and that PMMA is the only source of contamination. Last but not least, electrochemical delamination avoids the use of harsh chemicals, such as hydrochloric and sulfuric acid at high concentration. It is a good news to avoid doping or functionalization of graphene, which could severely downgrade the optical, electrical and mechanical properties of graphene [119].

\section{Water-assisted delamination}

Electrochemical delamination is the most widely studied method for the reuse and the recycle of the catalyst from graphene synthesized by CVD. However, the use of the electrolysis cell makes the process relatively complex. Researchers are looking for a more direct separation approach by modifying the CVD process to allow preservation of the catalyst under wet chemical separation. In a recent study, it was found that a prolonged contact of graphene/metal catalyst in pure water could facilitate graphene delamination. Water molecules could intercalate at the graphene/metal interface for oxidation of the catalyst. Although graphene is always referred as a highly hydrophobic material, it was experimentally shown that physisorption of water on the graphene plane is possible; the number of water molecules adsorbed increases with temperature. Above $400{ }^{\circ} \mathrm{C}$, chemisorption would take place causing irreversible defects on the graphene plane [120]. The main difference between direct delamination discussed in the current section and conventional mechanical exfoliation of graphene discussed previously is the occurrence of intercalation of foreign molecules in the interspace of graphene/metal catalyst. This intercalation phenomenon leads to weaken the coupling between graphene and metal catalyst and subsequently facilitates the peeling step minimizing the mechanical damaging. Such method was shown to be efficient with $\mathrm{Cu}$ as catalyst and a direct ultra-sonication induces an easy peeling off graphene [121]. 
The direct contact of $\mathrm{Cu}$ with moisture and air could induce oxidation of $\mathrm{Cu}$ to cuprous oxide formation at the foil surface. As discussed in electrochemical delamination section, the formation of copper oxide is welcomed here to weaken the coupling between graphene and $\mathrm{Cu}$ regarding both chemical and mechanical interactions. Copper oxide henceforward allows an easier delamination of graphene from $\mathrm{Cu}$ even without electrochemically-induced bubbles [101, 102]. In high humidity environment, $\mathrm{Cu}$ will first be oxidized to $\mathrm{Cu}(\mathrm{OH})_{2}$. The $\mathrm{Cu}(\mathrm{OH})_{2}$ subsequently oxidize to $\mathrm{CuO}$ or $\mathrm{Cu}_{2} \mathrm{O}$ with releasing water and small amount of oxygen gas, where the oxygen gas further assists the delamination of graphene from copper [122]. This water-mediated graphene separation technique is a mild separation process that was mainly proved to be efficient to delaminate graphene from copper since copper is easily oxidized under ambient atmosphere. At the opposite side from graphene, $\mathrm{Cu}_{2} \mathrm{O}$ can be formed by exposure to ambient atmosphere and oxygen penetrates to the $\mathrm{Cu} /$ graphene interface, and subsequent immersion of the oxidized copper foil/graphene in mild acid facilitates a selective etching of the catalyst foil for graphene separation [123].

The main weakness of this approach is the slow process of $\mathrm{Cu}$ oxidation under ambient conditions. Graphene itself is chemically stable and widely believed to be impermeable to oxygen and it acts as a protective layer against $\mathrm{Cu}$ oxidation. $\mathrm{Cu}$ surface protected by graphene cannot be oxidized by air even under elevated temperature $\left(200{ }^{\circ} \mathrm{C}, 4 \mathrm{~h}\right)$; cuprous oxide was only detected at the defected areas of graphene, where air could penetrate through the cracks of graphene [124]. However, there is another view on the formation of copper oxide. Graphene was reported to play the role as "catalyst" in the oxidation of $\mathrm{Cu}$ foil. It was observed that $\mathrm{Cu}$ near or below the edge of graphene were undergoing oxidation faster than any other area [125]. According to Luo et al., functionalization of graphene is the main reason for this phenomenon. 
The graphene edges and defected graphene are composed by carbon with dangling bonds and lower in stability. These carbon sites are easier to be functionalized under exposure to moisture or air to form functional groups, such as $-\mathrm{OH}$ and $-\mathrm{COOH}$. The oxygen containing functional groups could form $\mathrm{H}_{2} \mathrm{O}-\mathrm{OH}$ complexes, and thus facilitating the electron transfer between $\mathrm{H}_{2} \mathrm{O}$ $\mathrm{OH}$ complexes and the copper substrate, which accelerates oxidation of $\mathrm{Cu}$ near to the graphene edges [103]. Ferrighi et al. [126] providing another view of the interaction between water, graphene and copper based on Density Functional Theory (DFT) simulation. The authors observe the formation of different functional group at the edge of graphene. The dissociation of energy of water molecule at unsaturated carbon atom at graphene edge strong enough to break the organometallic bonds of carbon and $\mathrm{Cu}$, to form one ether $(-\mathrm{C}-\mathrm{O}-\mathrm{C}-)$ and one $\mathrm{CH}_{2}$ group.

The intercalation of oxygen molecule between graphene flake and $\mathrm{Cu}$ could be observed under months of exposure in air. The charge transfer between $\mathrm{Cu}$ and graphene is annulled by the intercalation of oxygen, resulting the graphene has the similar properties as free-standing graphene. Blume et al. [127] claimed that the oxygen intercalation initial induces the formation of $\mathrm{Cu}$ sub-oxide state or adsorbate-like copper oxide, instead of copper oxide. The intercalated oxygen can be removed with annealing in vacuum $\left(200-300{ }^{\circ} \mathrm{C}\right)$ without giving damage to graphene and $\mathrm{Cu}$. However, $\mathrm{Cu}$ sub-oxide state or adsorbate-like copper oxide will transform to copper oxide under longer exposure to air.

In water or under high humidity, the rate of oxidation of copper was shown to increase especially under heating [128]. Hydrophilicity of cuprous oxide is believed to play the role for favoring diffusion of water molecules at the interface between $\mathrm{Cu}$ and graphene [129]. However, decoupling of graphene from $\mathrm{Cu}$ with cuprous oxide alone is not sufficient to balance the adhesion forces between graphene and $\mathrm{Cu}$. Normally, a PMMA coating is often used as a 
protective layer and graphene with reduced adhesion can be peeled off mechanically with adhesive tape. The oxidation of $\mathrm{Cu}$ surface was proven able accelerating $\mathrm{R} 2 \mathrm{R}$ process for graphene delamination; it was reported to delaminate much faster compared to the standard R2R process, as compared with conventional R2R process that requiring the etching of $\mathrm{Cu}$ foil [130]. It is mainly due to the avoidance of time consuming catalyst etching process. However, mechanical peeling inevitably creates large scale defects and it is the vital weakness for this approach [131].

Cao et al. [132] demonstrated a method to measure the adhesion force between graphene and $\mathrm{Cu}$. The method can be transformed into a modified water-assisted delamination of graphene from $\mathrm{Cu}$ substrate. A small hole with diameter of $1 \mathrm{~mm}$ was drilled through the $\mathrm{Cu}$ substrate, but without harming the graphene. Pressurized water (up to $120 \mathrm{kPa}$ ) water was pumped through the hole to apply pushing force to graphene sheet on copper. The pushing force will slowly delaminate the graphene from $\mathrm{Cu}$ substrate. The constant force applied will minimize the mechanical defect induced to the graphene sheet. This method will overcome the time consuming problem that faced by water assisted delamination.

Several approaches have been proposed to avoid the step of peeling proved to damage graphene. Van deer Laan et al. [133] reported that graphene grown from low temperature plasma-enhanced CVD could be delaminated easily by just dipping it into water. It was however reported that small amount of ethanol added into the water could reduce the surface tension of water and that it increases that way the wettability of graphene to facilitate its deposit on the chosen substrate. One of the drawback of plasma enhanced CVD is that functional groups could be found on graphene. To overcome it, a thermal treatment is required to remove the grafted groups and restore the crystallinity of graphene [134]. The formation of copper oxide could be 
accelerated by using warm and stronger basic oxidation agent $\left(1 \mathrm{M} \mathrm{NaOH}, 60{ }^{\circ} \mathrm{C}\right)$, which favors the self-delamination process within 2-3 hours, and it does not require any external force to peel off graphene from copper foil [135].

Delamination of graphene from $\mathrm{Ni}$ is much more complicated than from $\mathrm{Cu}$. $\mathrm{Ni}$ has higher resistivity to be oxidized under ambient conditions and the spacing between $\mathrm{Ni}$ and graphene is relatively small, forbidding foreign molecules to intercalate easily. Fast cooling after CVD could induce the formation of a layer of nickel carbide at the Ni foil surface. A partial etching mechanism was proposed to delaminate graphene from Ni. Mild etchant was utilized to partially etch $\mathrm{Ni}$ and break the coupling forces between $\mathrm{Ni}$ and graphene; separation of graphene from Ni occurs without help of an additional peeling process [77]. Water-assisted delamination could be also utilized to re-transfer graphene already deposited onto a silicon wafer. By soaking graphene/ $\mathrm{SiO}_{2}$ into water, the water molecules intercalate between graphene and $\mathrm{SiO}_{2}[136]$. The driving force for graphene lift-off is mainly the capillary forces induced by water at the hydrophilic surface of $\mathrm{SiO}_{2}$, while the hydrophobic nature of graphene allows it to lift off from the substrate [137].

Water-assisted delamination process is a green and chemical-free process involving neither waste nor hazardous chemicals. It is the easiest separation process that has been reported so far. Furthermore, the re-transfer of graphene from a substrate to another has also been demonstrated. Nevertheless, it is a time-consuming process; hours or days are necessary until sufficient cuprous oxide could form at the interface of graphene and $\mathrm{Cu}$. And water or air only assists in weaken the coupling between graphene and $\mathrm{Cu}$ and subsequent mechanical peeling is required to complete separation. Large scale of tears and voids on the graphene sheet are difficult to avoid. The oxidation process has been reported to result in corrugating the surface of the $\mathrm{Cu}$ 
foil; the subsequent reduction process does not guarantee full recovery of its initial flatness. The morphology and the uniformity of graphene totally depend of the topography of the catalyst surface and a corrugated surface is shown to cause deviation in repeatability of subsequent CVD growth. Instead of water, it has been demonstrated by Ma et al. that CO can be intercalated between graphene and the metal catalyst foil and it weakens the interactions between them [138]. However, graphene was observed to be broken in pieces after its subsequent transfer in PDMS stamp. The main difficulty of this method is to apply the adhesive material uniformly throughout the surface of graphene and peeling it off with a minimum of damages. Large organic molecules such as 1-octanethiol possess the ability to form a self-assembled monolayer (SAM) on the metal surface because of the strong interaction between the sulfur atoms and the metal atoms. It could hence easily intercalate in between graphene and $\mathrm{Cu}$ and helps to peel off graphene. However, sodium hydroxide and hydrogen peroxide are required to remove 1-octanethiol from graphene after separation which is a quite tedious and time-consuming process and the quality of graphene could be degraded [139]. Last but not least, the water-based delaminate method is mainly reported to efficiently separate graphene from $\mathrm{Cu}$ due to the relatively large spacing between graphene and $\mathrm{Cu}$ and its high affinity to be oxidized. Nevertheless, the same approach is not relevant to be applied for the separation of graphene from $\mathrm{Ni}, \mathrm{Ru}$ and etc.

\section{Future prospect}

There are various approaches that have been proved to separate graphene from its respective metal catalyst without scarification of the catalyst. However, majority of the articles discussed in the current review do not focus their study towards the reuse of the catalyst. The 
main obstacle to overcome is the preservation or a recovery of a smooth catalyst surface after separation. The uniformity of graphene, in term of continuity of the number of layers is highly dependent on the surface morphology of the catalyst. Any protrusion on the metal surface results in encapsulation of metal particles by the graphenic materials contaminating the graphene surface; and such contaminants are very difficult to remove [77]. Furthermore, the small dented areas, especially at the grain boundary, are known to cause accumulation of carbon adatoms, and form graphene with a higher number of layers. On the other hand, majority of the articles reported on the graphene transfer processes that allow recycling the catalyst are focused on $\mathrm{Cu}$ and Pt. Other catalysts such as $\mathrm{Ni}, \mathrm{Ru}$, Ir, are rarely studied for that purpose. $\mathrm{Ni}$ and $\mathrm{Ru}$ that have a relatively strong coupling forces with graphene result in very small spacing between graphene and metal substrate to allow an effective intercalation of any foreign atoms or molecules. This problem is more severe if the graphene is grown through sublimation of $\mathrm{SiC}$ since coupling forces are much stronger between graphene and $\mathrm{SiC}$. This makes the separation of graphene from SiC almost impossible. More reliable separation methods are urgently required for the separation of graphene from $\mathrm{SiC}$.

So far, only the works reported by Nanjegowda et al. [130] and Deng et al. [99] with the $\mathrm{R} 2 \mathrm{R}$ separation approach involves a continuous process on graphene growth and separation, which has the potential to be applied for mass production. Deterioration of the graphene basal plane during mechanical peeling is the main problem that needs to overcome. Another method allowing to recycle the catalyst by means of electroplating was proposed by Kwon et al. The dissolved $\mathrm{Cu}$ foil during wet chemical etching could be used again to form new $\mathrm{Cu}$ film through electroplating and subsequent CVD for graphene formation [140]. However, the report on such method is very rare in literature. Boscá et al. designed an automatic graphene transfer system 
which is feasible to etch the $\mathrm{Cu}$ catalyst and deposit graphene onto silicon automatically [141]. This process could be further improved with electrochemical delamination or water-assisted delamination to obtain another route adaptable for industrial applications.

CVD synthesis can be modified in order to make the subsequent transfer process simpler. The conventional production of graphene by CVD always requires a temperature greater than $800^{\circ} \mathrm{C}$ allowing only certain substrates, such as silicon, quartz or alumina which can withstand this temperature range. To use a polymer, the graphene formation temperature can be lowered by using plasma assistance during graphene CVD growth. For example, a $\mathrm{Cu}$ film can be directly coated on a Polyimide (PI) layer [142] to grow graphene through plasma enhanced CVD. The growth temperature could be as low as $240{ }^{\circ} \mathrm{C}$ [143]. The graphene was deposited onto the polymer substrate after it was immersed in the etching solution. However, in that case, the continuous bombardment of plasma during CVD degraded the already grown graphene.

There are a large number of applications of graphene, and more are expected to be discovered in the near future. The mass production or continuous production of graphene will be needed to fulfill the market demand. The transfer of graphene needs further improvements to meet the production rate of graphene. R2R graphene synthesis and separation could be adaptable for industrial scale graphene production. Preservation and recycling of the metal catalyst would be major breakthroughs to reduce the cost of graphene production. In other words, the discovery of a method to produce free standing graphene under a continuous process and the realization of the reuse of catalyst is the main objective for mass scale production. And preservation of catalyst during separation is definitely one of the key steps to lower the graphene production cost especially regarding the cost of catalyst itself and the chemical waste treatments. Meanwhile, because of the increasing demand for foldable touch screen displays, transfer and proper 
placement of graphene on soft substrates are the next challenges. Placing large graphene sheets tightly onto uneven surfaces is still yet to be achieved.

\section{Summary}

It is over a decade since graphene revolution has started, and it is found to have the potential to be applied in various applications. To date, there is still a lot improvement required, especially on the transfer of wafer-scale graphene to arbitrary substrate. Preservation of the catalyst during the transfer process is still remaining as a challenging task for researchers around the world. Mechanical delamination is an easy and straightforward technique but the transferred graphene sheets suffer large scale of mechanical defects. Although it could be reduced by applying water-assisted delamination but the amount of defects is still significant. Among all the methods available now, "bubbling" delamination is reported to be the most promising approach to allow the reuse of catalyst by minimizing the introduced defects on the transferred graphene. The avoidance of defects and contamination on the transferred graphene is the main challenge in near future. For graphene commercialization, development of continuous graphene production and separation without destroying the catalyst could be obtained with the R2R process. However, improvements are required to minimize the defects on graphene.

\section{Acknowledgements}

The authors gratefully acknowledge the financial support provided by Universiti Sains Malaysia (USM Fellowship), Les Bourses du Gouvernement Français and the Fundamental Research Grant Scheme (FRGS) (Rising Star) (203/PJKIMIA/6071335).

\section{References}


[1] A. Bianco, H.-M. Cheng, T. Enoki, Y. Gogotsi, R. H. Hurt, N. Koratkar, T. Kyotani, M. Monthioux, C. R. Park, J. M. D. Tascon, J. Zhang, Carbon 2013, 65, 1.

[2] K. S. Novoselov, A. K. Geim, S. V. Morozov, D. Jiang, Y. Zhang, S. V. Dubonos, I. V. Grigorieva, A. A. Firsov, Science 2004, 306, 666.

[3] A. K. Geim, K. S. Novoselov, Nat. Mater. 2007, 6, 183.

[4] Y. T. Liang, M. C. Hersam, Journal of the American Chemical Society 2010, 132, 17661.

[5] C.-M. Seah, B. Vigolo, S.-P. Chai, A. R. Mohamed, Carbon 2016, 105, 496.

[6] W. S. Hummers, R. E. Offeman, J. Am. Chem. Soc. 1958, 80, 1339.

[7] J. Robinson, X. Weng, K. Trumbull, R. Cavalero, M. Wetherington, E. Frantz, M. LaBella, Z. Hughes, M. Fanton, D. Snyder, ACS Nano 2009, 4, 153.

[8] K. S. Kim, Y. Zhao, H. Jang, S. Y. Lee, J. M. Kim, K. S. Kim, J.-H. Ahn, P. Kim, J.-Y. Choi, B. H. Hong, Nature 2009, 457, 706.

[9] X. Li, W. Cai, J. An, S. Kim, J. Nah, D. Yang, R. Piner, A. Velamakanni, I. Jung, E. Tutuc, S. K. Banerjee, L. Colombo, R. S. Ruoff, Science 2009, 324, 1312.

[10] C.-M. Seah, S.-P. Chai, A. Mohamed, Carbon 2014, 70, 1.

[11] A. H. Castro Neto, F. Guinea, N. M. R. Peres, K. S. Novoselov, A. K. Geim, Rev. Mod. Phys. 2009, 81, 109.

[12] M. Ishigami, J. H. Chen, W. G. Cullen, M. S. Fuhrer, E. D. Williams, Nano Lett. 2007, 7, 1643.

[13] V. Geringer, M. Liebmann, T. Echtermeyer, S. Runte, M. Schmidt, R. RÃ¹/4ckamp, M. C. Lemme, M. Morgenstern, Phys. Rev. Lett. 2009, 102, 076102.

[14] F. Varchon, R. Feng, J. Hass, X. Li, B. N. Nguyen, C. Naud, P. Mallet, J. Y. Veuillen, C. Berger, E. H. Conrad, L. Magaud, Phys. Rev. Lett. 2007, 99, 126805. 
[15] G. Nicotra, Q. M. Ramasse, I. Deretzis, A. La Magna, C. Spinella, F. Giannazzo, ACS Nano 2013, 7, 3045.

[16] T. Ohta, A. Bostwick, T. Seyller, K. Horn, E. Rotenberg, Science 2006, 313, 951.

[17] C. Riedl, C. Coletti, U. Starke, J. Phys. D: Appl. Phys 2010, 43.

[18] S. Y. Zhou, G. H. Gweon, A. V. Fedorov, P. N. First, W. A. de Heer, D. H. Lee, F. Guinea, A. H. Castro Neto, A. Lanzara, Nat Mater 2007, 6, 770.

[19] J. Hass, F. Varchon, J. E. Millán-Otoya, M. Sprinkle, N. Sharma, W. A. de Heer, C. Berger, P. N. First, L. Magaud, E. H. Conrad, Phys. Rev. Lett 2008, 100, 125504.

[20] J. Jobst, D. Waldmann, F. Speck, R. Hirner, D. K. Maude, T. Seyller, H. B. Weber, Phys. Rev, B 2010, 81, 195434.

[21] J. L. Tedesco, B. L. VanMil, R. L. Myers-Ward, J. M. McCrate, S. A. Kitt, P. M. Campbell, G. G. Jernigan, J. C. Culbertson, J. C. R. Eddy, D. K. Gaskill, Appl. Phys. Lett. 2009, $95,122102$.

[22] V. Panchal, K. Cedergren, R. Yakimova, A. Tzalenchuk, S. Kubatkin, O. Kazakova, J. Appl. Phys. 2012, 111, 07E509.

[23] R. S. Shishir, D. K. Ferry, J. Phys. Conden. Matter 2009, 21, 232204.

[24] S. Weingart, C. Bock, U. Kunze, K. V. Emtsev, T. Seyller, L. Ley, Physica E Low Dimens. Syst. Nanostruct. 2010, 42, 687.

[25] A. Tzalenchuk, S. Lara-Avila, A. Kalaboukhov, S. Paolillo, M. Syvajarvi, R. Yakimova, O. Kazakova, T. J. B. M. Janssen, V. Fal'ko, S. Kubatkin, Nat Nano 2010, 5, 186.

[26] K. I. Bolotin, K. J. Sikes, Z. Jiang, M. Klima, G. Fudenberg, J. Hone, P. Kim, H. L. Stormer, Solid State Commun. 2008, 146, 351. 
[27] J. H. Chen, M. Ishigami, C. Jang, D. R. Hines, M. S. Fuhrer, E. D. Williams, Adv. Mater. 2007, 19, 3623.

[28] S. Sonde, C. Vecchio, F. Giannazzo, R. Yakimova, V. Raineri, E. Rimini, Physica E Low Dimens. Syst. Nanostruct. 2012, 44, 993.

[29] C. Enderlein, Y. S. Kim, A. Bostwick, E. Rotenberg, K. Horn, New J. Phys. 2010, 12.

[30] E. Sutter, D. P. Acharya, J. T. Sadowski, P. Sutter, Appl. Phys. Lett. 2009, 94, 133101.

[31] J. Sun, J. B. Hannon, R. M. Tromp, P. Johari, A. A. Bol, V. B. Shenoy, K. Pohl, ACS Nano 2010, 4, 7073.

[32] Y. S. Dedkov, M. Fonin, New J. Phys. 12, 125004.

[33] M. Weser, Y. Rehder, K. Horn, M. Sicot, M. Fonin, A. B. Preobrajenski, E. N. Voloshina, E. Goering, Y. S. Dedkov, Appl. Phys. Lett. 2010, 96, 012504.

[34] Q. M. Ramasse, R. Zan, U. Bangert, D. W. Boukhvalov, Y.-W. Son, K. S. Novoselov, ACS Nano 2012, 6, 4063.

[35] D. W. Boukhvalov, M. I. Katsnelson, Appl. Phys. Lett. 2009, 95, 023109.

[36] Q. Ran, M. Gao, X. Guan, Y. Wang, Z. Yu, Appl. Phys. Lett. 2009, 94, 103511.

[37] M. Beshkova, L. Hultman, R. Yakimova, Vacuum 2016, 128, 186.

[38] G. Giovannetti, P. A. Khomyakov, G. Brocks, V. M. Karpan, J. van den Brink, P. J. Kelly, Phys. Rev. Lett. 2008, 101, 026803.

[39] A. Politano, A. R. Marino, V. Formoso, G. Chiarello, Carbon 2012, 50, 734.

[40] R. Addou, A. Dahal, M. Batzill, Nat. Nano 2013, 8, 41.

[41] C. R. Dean, A. F. Young, MericI, LeeC, WangL, SorgenfreiS, WatanabeK, TaniguchiT, KimP, K. L. Shepard, HoneJ, Nat. Nano 2010, 5, 722.

[42] R. T. Weitz, A. Yacoby, Nat. Nano 2010, 5, 699. 
[43] V. Verma, S. Das, I. Lahiri, W. Choi, Appl. Phys. Lett. 2010, 96, 203108.

[44] F. Cavallo, R. Rojas Delgado, M. M. Kelly, J. R. Sánchez Pérez, D. P. Schroeder, H. G. Xing, M. A. Eriksson, M. G. Lagally, ACS Nano 2014, 8, 10237.

[45] A. Ambrosi, M. Pumera, Nanoscale 2014, 6, 472.

[46] M. He, S. Novak, L. Vanamurthy, H. Bakhru, J. Plawsky, T.-M. Lu, Appl. Phys. Lett. 2010, 97, 252901.

[47] D. S. Lee, C. Riedl, B. Krauss, K. von Klitzing, U. Starke, J. H. Smet, Nano Lett. 2008, 8, 4320.

[48] J. Kim, H. Park, J. B. Hannon, S. W. Bedell, K. Fogel, D. K. Sadana, C. Dimitrakopoulos, Science 2013, 342, 833.

[49] T. Yoon, W. C. Shin, T. Y. Kim, J. H. Mun, T. S. Kim, B. J. Cho, Nano Lett. 2012, 12, 1448.

[50] J. Lahiri, T. S. Miller, A. J. Ross, L. Adamska, I. I. Oleynik, M. Batzill, New J. Phys. 2011, 13

[51] J.-H. Lee, E. K. Lee, W.-J. Joo, Y. Jang, B.-S. Kim, J. Y. Lim, S.-H. Choi, S. J. Ahn, J. R. Ahn, M.-H. Park, C.-W. Yang, B. L. Choi, S.-W. Hwang, D. Whang, Science 2014, 344, 286.

[52] Y. Zhang, L. Zhang, C. Zhou, Acc. Chem. Res. 2013, 46, 2329.

[53] J. Kang, D. Shin, S. Bae, B. H. Hong, Nanoscale 2012, 4, 5527.

[54] L. Banszerus, M. Schmitz, S. Engels, J. Dauber, M. Oellers, F. Haupt, K. Watanabe, T. Taniguchi, B. Beschoten, C. Stampfer, Sci. Adv. 2015, 1, e1500222.

[55] E. H. Lock, S. C. Hernandez, T. J. Anderson, S. W. Schmucker, M. Laskoski, S. P. Mulvaney, F. J. Bezares, J. D. Caldwell, P. E. Sheehan, J. T. Robinson, B. N. Feygelson, S. G. Walton, Surf. Coat. Technol. 2013. 
[56] S. Unarunotai, J. C. Koepke, C.-L. Tsai, F. Du, C. E. Chialvo, Y. Murata, R. Haasch, I. Petrov, N. Mason, M. Shim, J. Lyding, J. A. Rogers, ACS Nano 2010, 4, 5591.

[57] C. Kim, J. Y. Woo, J. Choi, J. Park, C.-S. Han, Scr. Mater. 2012, 66, 535.

[58] Y. Kwanghyun, Y. Takei, H. Bo, S. Chiashi, K. Matsumoto, I. Shimoyama, "Direct physical exfoliation and transfer of graphene grown via ethanol chemical vapor deposition", presented at Micro Electro Mechanical Systems (MEMS), 2011 IEEE 24th International Conference on, 23-27 Jan. 2011, 2011.

[59] C. Y. Su, D. Fu, A. Y. Lu, K. K. Liu, Y. Xu, Z. Y. Juang, L. J. Li, Nanotechnology 2011, $22,185309$.

[60] M. J. Allen, V. C. Tung, L. Gomez, Z. Xu, L. M. Chen, K. S. Nelson, C. Zhou, R. B. Kaner, Y. Yang, Adv. Mater. 2009, 21, 2098.

[61] J.-Y. Choi, Nat. Nano 2013, 8, 311.

[62] L.-H. Liu, M. Yan, Nano Lett. 2009, 9, 3375.

[63] E. H. Lock, M. Baraket, M. Laskoski, S. P. Mulvaney, W. K. Lee, P. E. Sheehan, D. R. Hines, J. T. Robinson, J. Tosado, M. S. Fuhrer, S. C. Hernández, S. G. Walton, Nano Lett. 2012, $12,102$.

[64] S. Bae, H. Kim, Y. Lee, X. Xu, J.-S. Park, Y. Zheng, J. Balakrishnan, T. Lei, H. Ri Kim, Y. I. Song, Y.-J. Kim, K. S. Kim, B. Ozyilmaz, J.-H. Ahn, B. H. Hong, S. Iijima, Nat. Nano 2010, 5, 574 .

[65] T. Yamada, M. Ishihara, J. Kim, M. Hasegawa, S. Iijima, Carbon 2012, 50, 2615.

[66] T. Hesjedal, Appl. Phys. Lett. 2011, 98, 133106.

[67] J. Kang, S. Hwang, J. H. Kim, M. H. Kim, J. Ryu, S. J. Seo, B. H. Hong, M. K. Kim, J.-B. Choi, ACS Nano 2012, 6, 5360. 
[68] M. Coroş, F. Pogăcean, M.-C. Roşu, C. Socaci, G. Borodi, L. Mageruşan, A. R. Biriş, S. Pruneanu, RSC Adv. 2016, 6, 2651.

[69] N. Liu, F. Luo, H. Wu, Y. Liu, C. Zhang, J. Chen, Adv. Func. Mater. 2008, 18, 1518.

[70] X. Yang, H. Peng, Q. Xie, Y. Zhou, Z. Liu, J. Electroanal. Chem. 2012.

[71] Y. H. Zhang, H. R. Zhang, B. Wang, Z. Y. Chen, Y. Q. Zhang, Y. P. Sui, B. Zhu, C. M. Tang, X. L. li, X. M. Xie, G. H. Yu, Z. Jin, X. Y. Liu, Appl. Phys. Lett. 2014, 104, 143110.

[72] S. Hu, M. Lozada-Hidalgo, F. Wang, A. Mishchenko, F. Schedin, R. Nair, E. Hill, D. Boukhvalov, M. Katsnelson, R. Dryfe, Nature 2014.

[73] Y. Wang, Y. Zheng, X. Xu, E. Dubuisson, Q. Bao, J. Lu, K. P. Loh, ACS Nano 2011, 5, 9927.

[74] L. Gao, W. Ren, H. Xu, L. Jin, Z. Wang, T. Ma, L.-P. Ma, Z. Zhang, Q. Fu, L.-M. Peng, X. Bao, H.-M. Cheng, Nat Commun 2012, 3, 699.

[75] Z. Zhan, J. Sun, L. Liu, E. Wang, Y. Cao, N. Lindvall, G. Skoblin, A. Yurgens, J. Mater. Chem. C 2015, 3, 8634.

[76] G. Fisichella, S. Di Franco, F. Roccaforte, S. Ravesi, F. Giannazzo, Appl. Phys. Lett. 2014, 104, 233105.

[77] C.-M. Seah, B. Vigolo, S.-P. Chai, S. Ichikawa, J. Gleize, F. Le Normand, F. Aweke, A. R. Mohamed, Carbon 2016, 96, 268.

[78] Z. Y. Xia, S. Pezzini, E. Treossi, G. Giambastiani, F. Corticelli, V. Morandi, A. Zanelli, V. Bellani, V. Palermo, Adv. Func. Mater. 2013, 23, 4684.

[79] L. Liu, X. Liu, Z. Zhan, W. Guo, C. Xu, J. Deng, D. Chakarov, P. Hyldgaard, E. Schröder, A. Yurgens, J. Sun, Adv. Mater. Interfaces 2015. 
[80] E. Koren, E. Sutter, S. Bliznakov, F. Ivars-Barcelo, P. Sutter, Appl. Phys. Lett. 2013, 103, 121602.

[81] K. Verguts, K. Schouteden, C.-H. Wu, L. Peters, N. Vrancken, X. Wu, Z. Li, M. Erkens, C. Porret, C. Huyghebaert, C. Van Haesendonck, S. De Gendt, S. Brems, ACS Appl. Mater. Interfaces 2017, 9, 37484 .

[82] I. Palacio, G. Otero-Irurueta, C. Alonso, J. Martínez, E. López-Elvira, I. Muñoz-Ochando, H. J. Salavagione, M. F. López, M. García-Hernández, J. Méndez, G. J. Ellis, J. A. Martín-Gago, Carbon 2018, 129, 837.

[83] D. L. Mafra, T. Ming, J. Kong, Nanoscale 2015, 7, 14807.

[84] M. Seifert, S. Drieschner, B. M. Blaschke, L. H. Hess, J. A. Garrido, Diam. Relat. Mater. 2014, 47, 46 .

[85] c. J. L. de la Rosa, J. Sun, N. Lindvall, M. T. Cole, Y. Nam, M. Löffler, E. Olsson, K. B. K. Teo, A. Yurgens, Appl. Phys. Lett. 2013, 102, 022101.

[86] S. Karamat, S. Sonuşen, Ü. Çelik, Y. Uysallı, A. Oral, Appl. Surf. Sci. 2016, 368, 157.

[87] T. Ciuk, I. Pasternak, A. Krajewska, J. Sobieski, P. Caban, J. Szmidt, W. Strupinski, J. Phys. Chem. C 2013, 117, 20833.

[88] J. Sun, X. Fan, W. Guo, L. Liu, X. Liu, J. Deng, C. Xu, Sensors 2015, 15, 31811.

[89] W. I. Lee, I. Y. Sohn, B. Y. Kim, Y. M. Son, J. H. Shin, H. M. Kim, Y. J. Suh, N.-E. Lee, Sci. Adv. Mater. 2015, 7, 1540.

[90] J. Sun, S. Deng, W. Guo, Z. Zhan, J. Deng, C. Xu, X. Fan, K. Xu, W. Guo, Y. Huang, J. Nanomater. 2016, 2016.

[91] X. Wang, L. Tao, Y. Hao, Z. Liu, H. Chou, I. Kholmanov, S. Chen, C. Tan, N. Jayant, Q. Yu, Small 2013, 10, 694. 
[92] Y. Fan, K. He, H. Tan, S. Speller, J. Warner, Chem. Mater. 2014, 264984

[93] J. Lee, Y. Kim, H.-J. Shin, C. Lee, D. Lee, S. Lee, C.-Y. Moon, S. U. Lee, S. J. kim, J. H.

Ji, H. S. Yoon, S. C. Jun, ACS Appl. Mater. Interfaces. 2014, 6, 12588.

[94] J. Lee, S. Lee, H. Yu, Coatings 2017, 7, 218.

[95] L. Shi, Y. Liu, F. Yang, L. Gao, J. Sun, Nanotechnology 2014, 25, 145704.

[96] L. Koefoed, M. Kongsfelt, S. Ulstrup, A. G. Čabo, A. Cassidy, P. R. Whelan, M. Bianchi, M. Dendzik, F. Pizzocchero, B. Jørgensen, J. Phys. D Appl. Phys. 2015, 48, 115306

[97] Z. Salmi, L. Koefoed, B. B. E. Jensen, A. G. Čabo, P. Hofmann, S. U. Pedersen, K. Daasbjerg, ChemElectroChem 2016, 3, 2202.

[98] I. Š. Rakić, D. Čapetab, M. Plodinec, M. Kralj, Carbon 2016, 96, 243.

[99] B. Deng, P.-C. Hsu, G. Chen, B. Chandrashekar, L. Liao, Z. Ayitimuda, J. Wu, Y. Guo, L. Lin, Y. Zhou, Nano Lett. 2015, 15, 4206.

[100] Z. Wang, Z. Liu, M. A. Monne, S. Wang, Q. Yu, M. Y. Chen, RSC Adv. 2016, 6, 24865.

[101] F. Pizzocchero, B. S. Jessen, P. R. Whelan, N. Kostesha, S. Lee, J. D. Buron, I. Petrushina, M. B. Larsen, P. Greenwood, W. J. Cha, K. Teo, P. U. Jepsen, J. Hone, P. Bøggild, T. J. Booth, Carbon 2015, 85, 397.

[102] C. T. Cherian, F. Giustiniano, I. Martinez-Hernandez, H. Andersen, J. Balakrishnan, B. Özyilmaz, Small 2014, 11, 189.

[103] D. Luo, X. You, B.-W. Li, X. Chen, H. J. Park, M. Jung, T. Y. Ko, K. Wong, M. Yousaf, X. Chen, M. Huang, S. H. Lee, Z. Lee, H.-J. Shin, S. Ryu, S. K. Kwak, N. Park, R. R. Bacsa, W. Bacsa, R. S. Ruoff, Chem. Mater. 2017, 29, 4546.

[104] M. C. Wang, W. P. Moestopo, S. Takekuma, S. F. Barna, R. T. Haasch, S. Nam, J. Mater. Chem. C 2017, 5, 11226. 
[105] Z. Zou, X. Song, K. Chen, Q. Ji, Y. Zhang, Z. Liu, Nano Res. 2015, 8, 592.

[106] S. Gorantla, A. Bachmatiuk, J. Hwang, H. A. Alsalman, J. Y. Kwak, T. Seyller, J. Eckert, M. G. Spencer, M. H. Rummeli, Nanoscale 2014, 6, 889.

[107] D. Zhang, Z. Jin, J. Shi, X. Wang, S. Peng, S. Wang, Chem. Commun. 2015, 51, 2987.

[108] J.-K. Choi, J. Kwak, S.-D. Park, H. D. Yun, S.-Y. Kim, M. Jung, S. Y. Kim, K. Park, S. Kang, S.-D. Kim, D.-Y. Park, D.-S. Lee, S.-K. Hong, H.-J. Shin, S.-Y. Kwon, ACS Nano 2015, 9, 679.

[109] L. B. Biedermann, T. E. Beechem, A. J. Ross, T. Ohta, S. W. Howell, New J. Phys. 2010, $12,125016$.

[110] D.-Y. Wang, I. S. Huang, P.-H. Ho, S.-S. Li, Y.-C. Yeh, D.-W. Wang, W.-L. Chen, Y.-Y. Lee, Y.-M. Chang, C.-C. Chen, C.-T. Liang, C.-W. Chen, Adv. Mater. 2013, 25, 4521.

[111] W. Jung, D. Kim, M. Lee, S. Kim, J. Kim, C. Han, Adv. Mater. 2014, 26, 6394.

[112] Z. Mutlu, I. Ruiz, R. J. Wu, R. Ionescu, S. Shahrezaei, S. Temiz, M. Ozkan, K. A. Mkhoyan, C. S. Ozkan, RSC Adv. 2017, 7, 32209.

[113] Y.-H. Lee, J.-H. Lee, Appl. Phys. Lett. 2010, 96, 083101.

[114] L. Chen, Z. Huang, S. Kumar, Appl. Phys. Lett. 2013, 103, 123110.

[115] P. Sutter, J. T. Sadowski, E. Sutter, Phys. Rev. B 2009, 80, 245411.

[116] Y. Gamo, A. Nagashima, M. Wakabayashi, M. Terai, C. Oshima, Surf. Sci. 1997, 374, 61.

[117] C. H. A. Wong, M. Pumera, J. Phys. Chem. C 2016.

[118] G. Lupina, J. Kitzmann, I. Costina, M. Lukosius, C. Wenger, A. Wolff, S. Vaziri, M. Östling, I. Pasternak, A. Krajewska, W. Strupinski, S. Kataria, A. Gahoi, M. C. Lemme, G. Ruhl, G. Zoth, O. Luxenhofer, W. Mehr, ACS Nano 2015, 9, 4776. 
[119] T. Ma, K. Miyazaki, H. Ariga, S. Takakusagi, K. Asakura, Bull. Chem. Soc. Jpn 2015, 88, 1029.

[120] N. M. Bom, G. V. Soares, M. H. de Oliveira Junior, J. M. J. Lopes, H. Riechert, C. Radtke, J. Phys. Chem. C 2016, 120, 201.

[121] C. Wu, F. Li, W. Wu, W. Chen, T. Guo, Appl. Phys. Lett. 2014, 105, 243509.

[122] P. R. Whelan, B. S. Jessen, R. Wang, B. Luo, A. C. Stoot, D. M. A. Mackenzie, P. Braeuninger-Weimer, A. Jouvray, L. Prager, L. Camilli, S. Hofmann, P. BØggild, T. J. Booth, Carbon 2017, 117, 75.

[123] A.-Y. Lu, S.-Y. Wei, C.-Y. Wu, Y. Hernandez, T.-Y. Chen, T.-H. Liu, C.-W. Pao, F.-R. Chen, L.-J. Li, Z.-Y. Juang, RSC Adv. 2012, 2, 3008.

[124] S. Chen, L. Brown, M. Levendorf, W. Cai, S.-Y. Ju, J. Edgeworth, X. Li, C. W. Magnuson, A. Velamakanni, R. D. Piner, ACS nano 2011, 5, 1321.

[125] B. Luo, P. R. Whelan, A. Shivayogimath, D. M. A. Mackenzie, P. BØggild, T. J. Booth, Chem. Mater. 2016, 28, 3789.

[126] L. Ferrighi, D. Perilli, D. Selli, C. Di Valentin, ACS Appl. Mater. Interfaces 2017, 9, 29932.

[127] R. Blume, P. R. Kidambi, B. C. Bayer, R. S. Weatherup, Z.-J. Wang, G. Weinberg, M.-G. Willinger, M. Greiner, S. Hofmann, A. Knop-Gericke, R. Schlogl, Phys. Chem. Chem. Phys. 2014, 16, 25989.

[128] R. Wu, L. Gan, X. Ou, Q. Zhang, Z. Luo, Carbon 2016, 98, 138.

[129] K. Tang, X. Wang, W. Yan, J. Yu, R. Xu, J. Membr. Sci. 2006, 286, 279.

[130] B. N. Chandrashekar, B. Deng, A. S. Smitha, Y. Chen, C. Tan, H. Zhang, H. Peng, Z. Liu, Adv. Mater. 2015, 27, 5210. 
[131] P. Gupta, P. Dongare, S. Grover, S. Dubey, H. Mamgain, A. Bhattacharya, M. Deshmukh, Sci. Rep. 2014, 4, 3882.

[132] Z. Cao, P. Wang, W. Gao, L. Tao, J. W. Suk, R. S. Ruoff, D. Akinwande, R. Huang, K. M. Liechti, Carbon 2014, 69, 390.

[133] T. van der Laan, S. Kumar, K. K. Ostrikov, Nanoscale 2015, 7, 20564.

[134] K. E. Whitener, W.-K. Lee, N. D. Bassim, R. Stroud, J. T. Robinson, P. E. Sheehan, Nano lett. 2016.

[135] R. Wang, P. R. Whelan, P. Braeuninger-Weimer, S. Tappertzhofen, J. A. AlexanderWebber, Z. A. Van Veldhoven, P. R. Kidambi, B. S. Jessen, T. Booth, P. BØggild, S. Hofmann, ACS Appl. Mater. Interfaces 2016, 8, 33072.

[136] D. Lee, G. Ahn, S. Ryu, J. Am. Chem. Soc. 2014, 136, 6634.

[137] G. F. Schneider, V. E. Calado, H. Zandbergen, L. M. K. Vandersypen, C. Dekker, Nano Lett. 2010, 10, 1912.

[138] D. Ma, Y. Zhang, M. Liu, Q. Ji, T. Gao, Y. Zhang, Z. Liu, Nano Res. 2013, 6, 671.

[139] M. Ohtomo, Y. Sekine, S. Wang, H. Hibino, H. Yamamoto, Nanoscale 2016.

[140] K. Kwon, J. Ham, S. Kim, J.-L. Lee, S. Kim, Sci. Rep. 2014, 4, 4830.

[141] A. Boscá, J. Pedrós, J. Martínez, T. Palacios, F. Calle, Sci. Rep. 2016, 6.

[142] Y. J. Kim, S. J. Kim, M. H. Jung, K. Y. Choi, S. Bae, S. K. Lee, Y. Lee, D. Shin, B. Lee, H. Shin, M. Choi, K. Park, J. H. Ahn, B. H. Hong, Nanotechnology 2012, 23.

[143] G. Kalita, K. Wakita, M. Umeno, RSC Adv. 2012, 2, 2815. 